PUBLICATION III

\title{
Adiabatic and multimode interference couplers on silicon-on-insulator
}

In: IEEE Photonics Technology Letters 2006.

Vol. 18, No. 21, pp. 2287-2289.

Reprinted with permission from the publisher.

(C) 2006 IEEE 


\title{
Adiabatic and Multimode Interference Couplers on Silicon-on-Insulator
}

\author{
Kimmo Solehmainen, Markku Kapulainen, Mikko Harjanne, and Timo Aalto
}

\begin{abstract}
Adiabatic and multimode interference (MMI) 3-dB couplers based on silicon-on-insulator rib waveguides were fabricated and measured. For testing purposes, pairs of identical couplers were cascaded to form Mach-Zehnder interferometers. The adiabatic couplers showed excess on-chip loss of $\sim 0.5 \mathrm{~dB}$, extinction ratio (ER) of 15-20 dB, and a wide spectral range. The MMI couplers processed on the same wafer showed similar loss per coupler, higher ER, and limited spectral characteristics.
\end{abstract}

Index Terms-Integrated optics, optical device fabrication, optical planar waveguide couplers, silicon-on-insulator (SOI) technology.

\section{INTRODUCTION}

$\mathbf{O}$ PTICAL couplers are used in photonic integrated circuits (PICs) when it is necessary to couple light between different waveguides. Traditionally, they were realized using directional couplers. Unfortunately, these components have been found to be sensitive to wavelength, fabrication tolerances, and polarization state of light. During the past ten years, they have mostly been replaced by multimode interference (MMI) couplers, which have relaxed fabrication tolerances and sufficiently low polarization dependency. However, MMI couplers still have rather large wavelength dependency. To circumvent these limitations, adiabatic couplers have been developed and tested on $\mathrm{LiNbO}_{3}$ [1], $\mathrm{SiO}_{2}$ [2], and more recently on polymeric [3] waveguide materials. The term adiabatic means that there is no energy change between different modes in the coupler structure. Thus, power coupled to the fundamental mode stays in the fundamental mode even in the presence of dimensional changes and additional modes. This principle distinguishes the adiabatic coupler from directional and MMI couplers, where the excitation of higher order modes is the main requirement for the operation. Unlike simple Y-junctions, adiabatic couplers can be used as passive $2 \times 2$ couplers and, thus, to construct, e.g., active $2 \times 2$ switches. The main drawback of previous adiabatic coupler demonstrations has been that in order to avoid the excitation of higher order modes, the adiabatic couplers have been significantly longer than the equivalent directional couplers or MMI couplers. Thus, adiabatic couplers have not been widely used in integrated optics.

Silicon-on-insulator (SOI) is an attractive platform for realizing PICs and for integrating them monolithically with control electronics. Due to the ultrahigh refractive index contrast, SOI

Manuscript received May 23, 2006; revised August 14, 2006.

The authors are with VTT Technical Research Centre of Finland, FI-02044 Espoo, Finland (e-mail: kimmo.solehmainen@vtt.fi; markku.kapulainen@vtt.fi; timo.aalto@vtt.fi; mikko.harjanne@vtt.fi).

Digital Object Identifier 10.1109/LPT.2006.885305

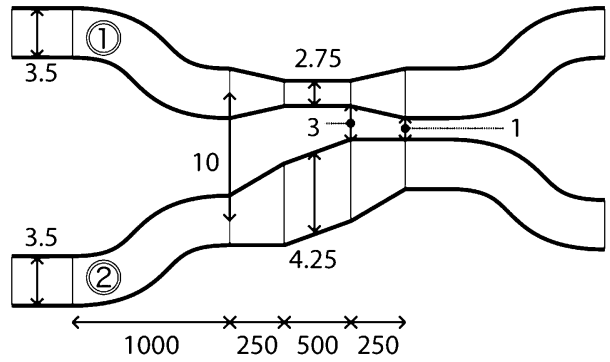

(a)

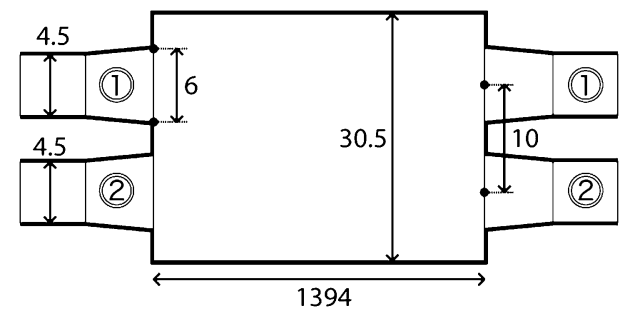

(b)

Fig. 1. Schematic view of (a) adiabatic coupler and (b) MMI coupler design. The dimensions are in micrometers (not in scale).

technology allows the large-scale integration of optical circuits. Extreme device miniaturization can be achieved with submicron nanowires [4], while mirrors and groove bends [5] enable the miniaturization of thicker waveguide devices as well. Compared with nanowires, rib waveguides with a larger cross section offer lower coupling losses to optical fibers and lasers, while maintaing single-moded (SM) operation.

In this study, SOI-based adiabatic couplers were, for the first time, fabricated and characterized using $4-\mu \mathrm{m}$-thick SM rib waveguides. Their performance was compared with MMI couplers, which, having been processed on the same SOI wafer, had identical fabrication tolerances. The properties under study included optical losses, polarization sensitivity, and spectral characteristics.

\section{DESIGN}

The design was based on a simple waveguide cross section analysis carried out with a commercial TempSelene software. The layout of a single adiabatic coupler is shown in Fig. 1(a). The width of the input and output waveguides of the adiabatic coupler is $3.5 \mu \mathrm{m}$. From the initial (axial) separation of $50 \mu \mathrm{m}$, Waveguides 1 and 2 are brought to the axial separation of $10 \mu \mathrm{m}$ with S-bends. After the bends, the waveguides are changed asymmetrically, so that Waveguides 1 and 2 are tapered to 2.75 and $4.25 \mu \mathrm{m}$, respectively. Then they are brought to a (edge-to-edge) distance of $3 \mu \mathrm{m}$, after which the actual 
coupling section takes place. In this region, the waveguides are tapered to the same width of $3.5 \mu \mathrm{m}$ and brought still closer to each other, to the minimum distance of $1 \mu \mathrm{m}$. The total length of the interaction region including all tapered waveguide sections is $1 \mathrm{~mm}$. After the coupling section, the waveguides are brought apart with S-bends. The 3.5- $\mu$ m-wide input and output waveguides of the couplers are tapered to the width of $8 \mu \mathrm{m}$ at the output to reduce the fiber-coupling losses of the test chip (not shown in Fig. 1).

In fully adiabatic operation, the optical power from the input port of Waveguide 2 excites the even system mode of the coupler and splits evenly to the two output waveguides (with the same phase). Similarly, optical power from the input port of Waveguide 1 excites the odd system mode and splits evenly to the output waveguides (with opposite phases). In order to enable easy and accurate characterization of the couplers, a pair of these 3 -dB couplers was cascaded to form a $2 \times 2$ Mach-Zehnder interferometer (MZI) device. The couplers are otherwise identical, but the latter one is point-mirrored from the first one. Thus, Waveguide 1 is made thicker and Waveguide 2 thinner in the latter coupling section. The MZI test device does not include any phase modulation structures, so that light coupled to Input 1 should always be transmitted to Output 2, and vice versa. High extinction ratio (ER) between the output powers of the two MZI outputs indicates that each coupler acts as a 3-dB coupler. Lower $\mathrm{ER}$ is an indication of unbalanced coupling, caused by the finite excitation of higher order modes.

The layout of an MMI coupler is shown in Fig. 1(b). The MMI tested here is based on paired interference, in which only some of the modes in the MMI region are excited [6]. In the layout, the length and width of the MMI region are 1394 and $30.5 \mu \mathrm{m}$, respectively. The input and output waveguides of the MMI structures are $4.5 \mu \mathrm{m}$ wide.

Before the connection to the MMI region, they are tapered from 4.5 to $6 \mu \mathrm{m}$ in order to avoid the excitation of higher order vertical modes. The axial separation of the input and output waveguides is $10 \mu \mathrm{m}$. As with the adiabatic couplers, a pair of identical couplers was used to form a $2 \times 2$ MZI layout.

When comparing the two design layouts, it can be seen that the adiabatic couplers are actually somewhat shorter than the equivalent MMI couplers. It was estimated that the used tapering angles are small enough to provide very adiabatic operation. However, longer tapers might behave even more adiabatically, i.e., to further suppress the excitation of any unwanted modes.

In order to measure the on-chip excess loss of the couplers, the mask design included also reference waveguides. They had a cross section identical to the input and output waveguides of the test devices. They also had the tapers and bends identical to the test devices, so that the loss originated from these sections did not contribute to the on-chip loss determination.

\section{FABRICATION AND MEASUREMENTS}

The base for the fabrication was an SOI wafer with $100-\mathrm{mm}$ diameter, 4.4- $\mu \mathrm{m}$ SOI layer, and 1- $\mu \mathrm{m}$-thick buried oxide layer. First, a 1- $\mu$ m-thick oxide hard mask was deposited on the wafer. It was then patterned with standard photolithography and etched using a parallel-plate reactive ion etcher. The silicon etch was carried out with an inductively coupled plasma etcher by using
TABLE I

LOSS OF MZI DEVICES BASED ON ADIABATIC AND MMI COUPLERS (Two COUPLERS CASCADED)

\begin{tabular}{ccccc}
\hline \hline & \multicolumn{4}{c}{ Excess On-Chip Loss at $1550 \mathrm{~nm}(\mathrm{~dB})$} \\
\cline { 2 - 5 } Measurement & $1_{\text {in }} \rightarrow 1_{\text {out }}$ & $1_{\text {in }} \rightarrow 2_{\text {out }}$ & $2_{\text {in }} \rightarrow 1_{\text {out }}$ & $2_{\text {in }} \rightarrow 2_{\text {out }}$ \\
\hline Adiabatic TE & 14.8 & 0.4 & 1.1 & 16.6 \\
Adiabatic TM & 20.7 & 0.8 & 1.2 & 19.0 \\
\hline MMI TE & 21.5 & 1.0 & 2.1 & 23.2 \\
MMI TM & 29.8 & 0.3 & 0.6 & 27.3 \\
\hline
\end{tabular}

the patterned oxide as a hard mask. The etch recipe was a modification from the STS Advanced Silicon Etch, as described in [7]. The etch depth was $2 \mu \mathrm{m}$. After removing the oxide mask, a $1-\mu \mathrm{m}$-thick thermal oxide was grown on top of the patterned silicon structure to reduce the surface roughness. During the oxidation, the thickness of the SOI layer decreased to $4 \mu \mathrm{m}$. The oxidation also changed the linewidth, so that the total linewidth change due to the fabrication (oxidation and photolithography) was $1.2 \mu \mathrm{m}$. The thermal oxide was removed with wet etching in buffered hydrofluoric acid before the growth of the cladding oxide layer. The $1-\mu \mathrm{m}$-thick cladding oxide was deposited with the tetra-ethyl-ortho-silicate process in a low-pressure chemical vapor deposition furnace. The final steps of the fabrication process were the dicing of the wafer and polishing the chip facets to optical quality.

The loss measurements were measured with both TE and TM input polarizations. In the measurements, linearly polarized light at 1550-nm wavelength was coupled from a polarization-maintaining fiber into an input waveguide. The transmitted light from an identical output waveguide was coupled into an optical power meter via a single-mode fiber. The use of SM fibers guaranteed that the insertion loss (IL) was measured for the fundamental mode only. Index matching oil (refractive index $\approx 1.5$ ) was used to reduce the reflections at the waveguide facets. The total IL of all test devices was calculated by subtracting the power transmitted directly from the input fiber to the output fiber. In order to determine the on-chip excess loss of a coupler, the rather imprecise input and output coupling losses should be subtracted from the IL. To improve the measurement accuracy, the total IL measured for a test device was compared with an equally long and wide reference waveguide.

In the spectral measurements, unpolarized light from an optical source with a spectral range of 1250-1650 nm was coupled into the input waveguide with an SM fiber. The output light was coupled into another SM fiber and guided to a spectrum analyzer.

\section{RESUltS AND DiscUSSION}

The excess on-chip losses of MZI devices based on both adiabatic and MMI couplers are summarized in Table I at $1550-\mathrm{nm}$ wavelength. For the adiabatic couplers, the loss is about $0.2-0.6 \mathrm{~dB}$ per coupler for both polarizations, while the ER of the MZI device is about $15 \mathrm{~dB}$ for TE and almost $20 \mathrm{~dB}$ for TM. The fiber-coupling loss of the test chips is estimated to be $3.9 \mathrm{~dB} /$ facet, which includes both the modal coupling and reflection losses.

The excess loss per MMI coupler is $0.2-1.0 \mathrm{~dB}$ for both polarizations. As with the adiabatic couplers, the variation in the 


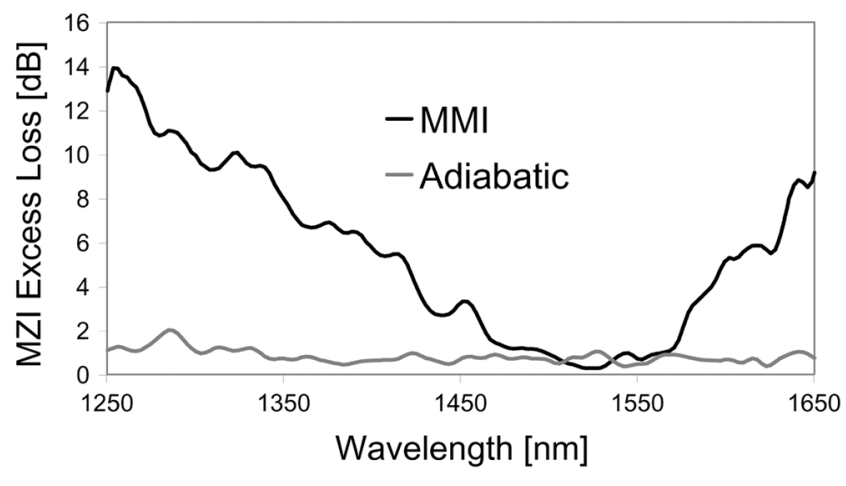

Fig. 2. Excess loss of the $2 \times 2$ MZI devices based on adiabatic and MMI couplers. The spectra are measured from input Port 1 to output Port 2.

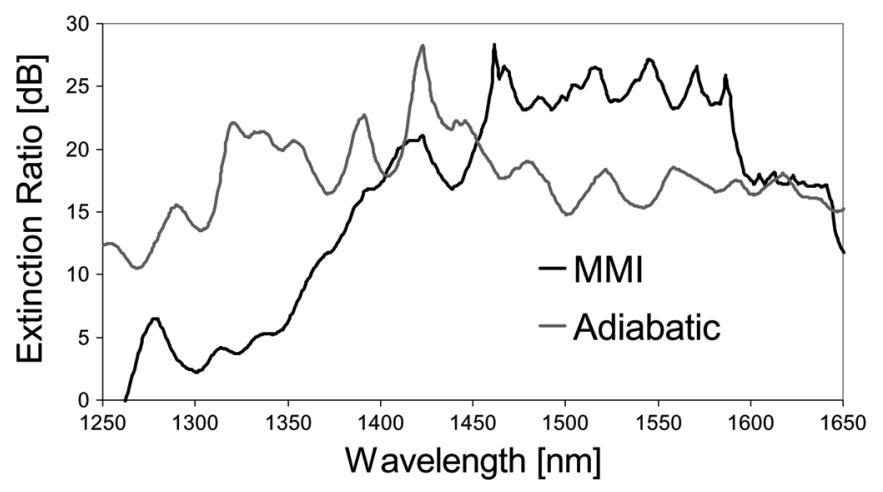

Fig. 3. ER of adiabatic and MMI couplers as a function of wavelength.

results is due to the finite accuracy of the measurement setup. The ER of the MMI-based MZI is over $20 \mathrm{~dB}$ for TE and almost $30 \mathrm{~dB}$ for TM, which is clearly better in comparison with the adiabatic coupler. The results of the MMI-based device are comparable to those achieved earlier with SOI [8]. In order to improve the ER of the adiabatic coupler-based device, the device geometry should be optimized to further suppress the residual excitation of the higher order modes.

The results from the spectral loss measurements are shown in Fig. 2. The MZI based on adiabatic couplers shows a very flat response with excess loss around $1 \mathrm{~dB}$ throughout the measured spectral range. The exception is the peak around $1290 \mathrm{~nm}$, where the maximum loss of $2 \mathrm{~dB}$ was measured. The MMI-based MZI shows losses about $1 \mathrm{~dB}$ in a spectral range of 1480-1570 nm. However, outside this low-loss region, the losses increase rapidly.

The spectral behavior of the ER for the MZI devices is shown in Fig. 3. For the MZI based on adiabatic couplers, the ER remains above $15 \mathrm{~dB}$ for the best part of the spectrum, except around $1270 \mathrm{~nm}$, where it reaches a minimum of $10 \mathrm{~dB}$. The fluctuations in the spectrum are caused by the residual excita- tion of the higher order modes, which could not be completely avoided in this first experiment. However, it can clearly be seen that the overall behavior of the ER spectrum is much flatter for the adiabatic couplers than for the MMI couplers.

Indeed, for the MMI-based MZI the ER decreases to zero at the wavelength of $1260 \mathrm{~nm}$. Only in the relatively narrow spectral range, near the targeted $1550-\mathrm{nm}$ wavelength, the MMI couplers show superior ER.

\section{CONCLUSION}

Adiabatic and MMI couplers on SOI were fabricated and characterized. The adiabatic coupler design was based on asymmetric coupler arms and 3-dB operation. The MMIs were based on paired interference and tapered input and output waveguides. In order to test their performance, a pair of identical couplers was cascaded to form a $2 \times 2$ MZI structure. The fabrication of the devices was based on single-mode $4-\mu$ m-thick rib waveguide technology.

In the measurements, the couplers based on different principles showed comparable losses and polarization sensitivities at $1550 \mathrm{~nm}$. However, the spectral range was clearly wider for the adiabatic couplers than for the MMI couplers. It was, therefore, shown that when a broad wavelength range is required, i.e., from 1300 to $1600 \mathrm{~nm}$, low-loss adiabatic couplers with acceptable ER can be realized on SOI. Furthermore, it was shown that using adiabatic couplers does not necessarily require larger component size than MMI couplers with comparable characteristics.

\section{REFERENCES}

[1] Y. Silberberg, P. Perlmuter, and J. E. Baran, "Digital optical switch," Appl. Phys. Lett., vol. 51, no. 16, pp. 1230-1232, Oct. 1987.

[2] R. Adar, C. H. Henry, R. F. Kazarinov, R. C. Kistler, and G. R. Weber, "Adiabatic 3-dB couplers, filters, and multiplexers made with silica waveguides on silicon," J. Lightw. Technol., vol. 10, no. 1, pp. 46-50, Jan. 1992.

[3] W. Yuan, S. Kim, W. H. Steier, and H. R. Fetterman, "Electrooptic polymeric digital optical switches (DOSs) with adiabatic couplers," IEEE Photon. Technol. Lett., vol. 17, no. 12, pp. 2568-2570, Dec. 2005.

[4] Y. A. Vlasov and S. J. McNab, "Losses in single-mode silicon-on-insulator strip waveguides and bends," Opt. Express, vol. 12, no. 8, pp. 1622-1631, Apr. 2004.

[5] K. Solehmainen, T. Aalto, J. Dekker, M. Kapulainen, M. Harjanne, and P. Heimala, "Development of multi-step processing in silicon-on-insulator for optical waveguide applications," J. Opt. A, Pure Appl. Opt., vol. 8, no. 7, pp. S455-S460, Jul. 2006.

[6] L. B. Soldano and E. C. M. Pennings, "Optical mode interference devices based on self-imaging: Principles and applications," J. Lightw. Technol., vol. 13, no. 4, pp. 615-627, Apr. 1995.

[7] K. Solehmainen, T. Aalto, J. Dekker, M. Kapulainen, M. Harjanne, K. Kukli, P. Heimala, K. Kolari, and M. Leskelä, "Dry-etched silicon-oninsulator waveguides with low propagation and fiber-coupling losses," J. Lightw. Technol., vol. 23, no. 11, pp. 3875-3880, Nov. 2005.

[8] T. Zinke, U. Fischer, B. Schüppert, and K. Peterman, "Theoretical and experimental investigation of optical couplers in SOI," in Proc. SPIE, Apr. 1997, vol. 3007, pp. 30-39. 